\title{
Euler Evolution for Singular Initial Data and Vortex Theory
}

\author{
C. Marchioro ${ }^{1, \star \star \star \star}$ and M. Pulvirenti ${ }^{2, \star}$
}

1 Dipartimento di Matematica, Università di Trento, I-38050 Povo, Trento, Italy

2 Dipartimento di Matematica, Università di Roma "La Sapienza," Piazzale A. Moro 5, I-00100 Roma, Italy

\begin{abstract}
We study the evolution of a two dimensional, incompressible, ideal fluid in a case in which the vorticity is concentrated in small, disjoint regions of the physical space. We prove, for short times, a connection between this evolution and the vortex model.
\end{abstract}

\section{Introduction}

In this paper we want to study some properties of the behaviour of a non-viscous, incompressible fluid in two dimensions. The Euler equations for the vorticity of such a fluid in all $\mathbb{R}^{2}$ are:

$$
\left.\begin{array}{l}
\frac{\partial}{\partial t} \omega(x, t)+(u \cdot \nabla) \omega(x, t)=0, \quad \nabla \cdot u=0, \\
\omega(x, t)=\operatorname{curl} u(x, t)=\left(\frac{\partial u_{2}}{\partial x_{1}}-\frac{\partial u_{1}}{\partial x_{2}}\right)(x, t), \quad x=\left(x_{1}, x_{2}\right) \in \mathbb{R}^{2}, \\
\omega(x, 0)=\omega_{0}(x) .
\end{array}\right\}
$$

Here $u=\left(u_{1}, u_{2}\right) \in \mathbb{R}^{2}$ denotes the velocity field.

If $u$ decays at infinity, the incompressibility condition allows us to reconstruct the velocity field by means of $\omega$. In fact, by $\nabla \cdot u=0$, there exists a function $\Psi$, such that $u=\nabla^{\perp} \Psi$, where $\nabla^{\perp}=\left(\frac{\partial}{\partial x_{2}},-\frac{\partial}{\partial x_{1}}\right)$. Hence $\Delta \Psi=-\omega$ and

$$
\begin{gathered}
u(x, t)=\int k(x-y) \omega(y) d y, \quad k=\nabla^{\perp} g, \\
g(r)=-\frac{1}{2 \pi} \ln |r| \quad r \in \mathbb{R}^{2} .
\end{gathered}
$$

* Partially supported by Italian C.N.R. (G.N.F.M.) and by Nato Grant No. 040.82

** Partially supported by NSF Grant No. PHY-8116101A01 
We consider now a situation in which the vorticity $\omega$ is initially concentrated in $N$ small regions of the physical space $\mathbb{R}^{2}$. To study this problem an idealized model, called the vortex model, has been introduced in the last century [1].

Consider the dynamics of $N$ points (called vortices) in $\mathbb{R}^{2}$, satisfying:

$$
\left.\begin{array}{l}
\dot{x}_{i}(t)=\sum_{\substack{j=1 \\
j \neq i}}^{N} a_{j} k\left(x_{i}(t)-x_{j}(t)\right), \quad a_{j} \in \mathbb{R}, j=1 \ldots N, \\
x_{i}(0)=x_{i} .
\end{array}\right\}
$$

Then, denoting by

$$
\omega_{0}(d x)=\sum_{i=1}^{N} a_{i} \delta_{x_{i}}(d x)
$$

the initial distribution of vorticity (here $\delta_{x_{i}}$ denotes the Dirac measure concentrated on $x_{i}$ ), the distribution

$$
\omega_{t}(d x)=\sum_{i=1}^{N} a_{i} \delta_{x_{i}(t)}(d x)
$$

would describe the vorticity at later times $t$.

If one believes the Euler equations to be the basic equations of the dynamics of an ideal fluid, a precise connection with the above particle model has to be established. The first natural step would be to investigate the Euler evolution given by (1.1) in some weak form, allowing signed measures, as (1.5), as the initial condition. As a consequence of (1.1) and a formal integration by parts, we get:

$$
\left.\begin{array}{l}
\frac{d}{d t} \omega_{t}(f)=\omega_{t}(u \cdot \nabla f) \\
\omega_{t}(f)=\int \omega_{t}(d x) f(x),
\end{array}\right\}
$$

where $u$ is given by (1.2) and $f$ is any smooth test function.

Unfortunately, due to the singularity of $k$ at the origin, $u=k * \omega$ makes sense only for absolutely continuous signed measures $\omega(d x)=\omega(x) d x$, with density $\omega$ in suitable $L_{p}\left(\mathbb{R}^{2}\right)$ spaces (i.e. $\omega \in L_{1} \cap L_{\infty}$ is a sufficient condition). Thus a direct connection between Euler Eqs. (1.7), (1.2), (1.3), and the vortex model (1.4) is impossible, unless one neglects the (infinite!) selfinteraction of a single vortex. In fact, defining the velocity field as

$$
u(x)=\int k(x-y) X(x \neq y) \omega(d y),
$$

where

$$
X(x \neq y)= \begin{cases}1 & \text { if } x \neq y \\ 0 & \text { otherwise }\end{cases}
$$

a direct computation shows (at least at a formal level) that the measure (1.6), obtained by the vortex motion (1.4), satisfies the modified Euler Eqs. (1.7) and (1.8).

Nevertheless, the question whether a more precise connection between the "true" Euler dynamics and the vortex motion can be established remains open. 
A reasonable approach, giving a justification of the vortex model, is the following. Suppose we have at time zero a vorticity distribution, with density $L_{1} \cap L_{\infty}$, arranged in $N$ small regions and approaching the initial distribution (1.5). Evolve it via the regular Euler equations. Then, at time $t$, such an evolved distribution is expected to approach the measure (1.6). If this is the case, such convergence would show that selfenergy is actually negligible.

In this paper we try to perform the above program, giving a proof of this feature for a single vortex in an external field in Sect. 2, and for $N$ vortices, for short times, in Sect. 3. Finally we briefly discuss the case when boundaries are present.

To conclude this section we notice that other connections between the Euler equations and the vortex motion are possible. It has been proved, under some technical hypothesis, that the vortex motion converges to the Euler dynamics, in the limit in which the number of vortices diverges and the intensity of each vortex vanishes in such a way that the vorticity profile approximates a smooth function [2]. For a review including also the case of nonvanishing viscosity, see [3].

\section{A Single Vortex in an External Field}

We consider a blob of vorticity moving according to the Euler equations in an external velocity field. Suppose

$$
\omega_{\varepsilon}(x, 0)=a \varepsilon^{-2} X_{\Lambda_{\varepsilon}}(x), \quad a>0
$$

is the initial distribution of vorticity, where $X_{A_{\varepsilon}}$ is the indicator of the open connected set $\Lambda_{\varepsilon}$, such that meas $\Lambda_{\varepsilon}=\varepsilon^{2}$.

Consider a given divergence-free, smooth, time-dependent velocity field $F(\cdot, t)$. A trajectory $x\left(t, x_{0}\right)$ of the particle of the fluid initially in $x_{0}$ satisfies:

$$
\left.\begin{array}{l}
\dot{x}^{\varepsilon}\left(t, x_{0}\right)=u\left(x^{\varepsilon}\left(t, x_{0}\right), t\right), \\
u_{\varepsilon}(x, t)=\int k(x-y) \omega_{\varepsilon}(y, t) d y+F(x, t), \\
x(0)=x_{0} .
\end{array}\right\}
$$

The vorticity is constant along the particle paths that are the characteristics of the Euler system. Therefore:

$$
\omega_{\varepsilon}(y, t)=\omega_{\varepsilon}\left(x^{\varepsilon}(-t, y), 0\right),
$$

and hence

$$
\omega_{\varepsilon}(y, t)=a \varepsilon^{-2} X_{\Lambda_{\varepsilon}^{t}}(x),
$$

where $\Lambda_{\varepsilon}^{t}$ is the evolution of $\Lambda_{\varepsilon}$ along the particle paths.

Because of divergence-free condition, the evolution (2.2) is Lebesgue measure preserving. In particular, meas $\Lambda_{\varepsilon}^{t}=$ meas $\Lambda_{\varepsilon}$. We notice that the evolution problem (2.2), (2.3) is uniquely solvable and this gives a unique weak solution of the Euler equations (1.1) with initial datum $\omega_{\varepsilon}$. 
We introduce the center of vorticity of the system, i.e.

$$
M_{\varepsilon}(t)=a^{-1} \int x \omega_{\varepsilon}(x, t) d x=a^{-1} \int x^{\varepsilon}(t, x) \omega_{\varepsilon}(x) d x,
$$

and we prove that in the limit $\varepsilon \rightarrow 0$, if $\Lambda_{\varepsilon}$ approachs the point $\bar{x}, M_{\varepsilon}(t)$ converges to $M(t)$, that is the particle path starting from $\bar{x}$ and generated by the external field $F$ only. This means that in this limit the blob does not influence the motion of the center of vorticity of the system.

More precisely:

Theorem 2.1. Let $\omega_{\varepsilon}, \varepsilon \in(0,1)$ be the sequence given by (2.1) and $f$ any continuous and bounded function $f: \mathbb{R}^{2} \rightarrow \mathbb{R}$. We suppose:

$$
\lim _{\varepsilon \rightarrow 0} \frac{1}{a} \int f(y) \omega_{\varepsilon}(y, 0) d y=f(\bar{x}), \quad \bar{x} \in \mathbb{R}^{2}, a>0 .
$$

Let $F(x, t): \mathbb{R}^{2} \times \mathbb{R} \rightarrow \mathbb{R}^{2}$ be a divergence-free vector field uniformly Lipschitz continuous in $x$, and continuous in $t$. Let $M_{\varepsilon}(t)$ be the center of vorticity of the blob $\omega_{\varepsilon}(\cdot, t)$, evolving according to (2.2) and (2.4). Then

$$
\lim _{\varepsilon \rightarrow 0} M_{\varepsilon}(t)=M(t), \quad t>0
$$

where

$$
M(t)=\bar{x}+\int_{0}^{t} d s F(M(s), s)
$$

Moreover

$$
\lim _{\varepsilon \rightarrow 0} \frac{1}{a} \int f(y) \omega_{\varepsilon}(y, t)=f(M(t)), \quad t>0 .
$$

Proof. First we observe that if $F \equiv 0, M_{\varepsilon}(t)=M_{\varepsilon}(0)$ is a constant of motion, as follows by direct computation. Hence the theorem holds. In general we have:

$$
\begin{aligned}
\frac{d}{d t} M_{\varepsilon}(t)= & a^{-1} \int \omega_{\varepsilon}(x) \dot{x}^{\varepsilon}(t, x) d x \\
= & a^{-1} \int \omega_{\varepsilon}(x) F\left(x^{\varepsilon}(t), t\right) d x \\
& {\left[\text { by symmetry } \int_{\Lambda_{t}^{\varepsilon}} d x \int_{\Lambda_{t}^{\varepsilon}} d y k(x-y)=0\right] } \\
= & a^{-1} \int \omega_{\varepsilon}(x, t) F(x, t) d x \\
& (\text { by the conservation of the measure }) \\
= & \varepsilon^{-2} \int_{\Lambda_{t}^{\varepsilon}} F(x, t) d x .
\end{aligned}
$$

Thus we have to prove that $\Lambda_{t}^{\varepsilon}$ is in a small neighborhood of the point $M_{\varepsilon}(t)$. To evaluate this fact we introduce the second moment

$$
\begin{aligned}
I_{\varepsilon}(t) & =\int\left(x-M_{\varepsilon}(t)\right)^{2} \omega_{\varepsilon}(x, t) d x \\
& =a \varepsilon^{-2} \int_{\Lambda^{\varepsilon}}\left(x^{\varepsilon}(t, x)-M_{\varepsilon}(t)\right)^{2} d x .
\end{aligned}
$$


Also $I_{\varepsilon}(t)$ would be a conserved quantity if $F \equiv 0$. In general

$$
\frac{d}{d t} I_{\varepsilon}(t)=2 a \varepsilon^{-2} \int_{\Lambda^{\varepsilon}}\left(x^{\varepsilon}(t, x)-M_{\varepsilon}(t)\right) \cdot F\left(x^{\varepsilon}(t, x), t\right) d x
$$

as follows by direct computation, since

$$
\int_{\Lambda_{t}^{\varepsilon}} d x \int_{\Lambda_{t}^{\varepsilon}} d y x \cdot k(x-y)=\frac{1}{2} \int_{\Lambda_{t}^{\varepsilon}} d x \int_{\Lambda_{t}^{\varepsilon}} d y(x-y) \cdot k(x-y)=0 .
$$

Hence, we denote by $L$ the Lipschitz constant of $F$,

$$
\left|\frac{d}{d t} I_{\varepsilon}(t)\right| \leqq 2 L I_{\varepsilon}(t)
$$

implying

$$
I_{\varepsilon}(t) \leqq I_{\varepsilon}(0) e^{2 L t} .
$$

Finally, for $t \in[0, T]$, using (2.10) and Cauchy-Schwarz inequality:

$$
\begin{aligned}
\left|M(t)-M_{\varepsilon}(t)\right| \leqq & \left|\bar{x}-M_{\varepsilon}(0)\right|+\int_{0}^{t} d s\left|F(M(s))-F\left(M_{\varepsilon}(s)\right)\right| \\
& +\int_{0}^{t} d s\left|F\left(M_{\varepsilon}(s)\right)-\varepsilon^{-2} \int_{\Lambda_{s}^{\varepsilon}} F(x, s) d x\right| \\
\leqq & \left|\bar{x}-M_{\varepsilon}(0)\right|+L \int_{0}^{t} d s\left|M(s)-M_{\varepsilon}(s)\right| \\
& +\varepsilon^{-2} L \int_{0}^{t} d s \int_{\Lambda_{s}^{\varepsilon}} d x\left|M_{\varepsilon}(s)-x\right| \\
\leqq & \left|\bar{x}-M_{\varepsilon}(0)\right|+\frac{L T}{\sqrt{a}} \sup _{t \in[0, T]} \sqrt{I_{\varepsilon}(t)} \\
& +L \int_{0}^{t} d s\left|M(s)-M_{\varepsilon}(s)\right| .
\end{aligned}
$$

By virtue of (2.6), $\lim _{\varepsilon \rightarrow 0}\left|\bar{x}-M_{\varepsilon}(0)\right|=\lim _{\varepsilon \rightarrow 0} I_{\varepsilon}(0)=0$, implying by (2.14) that the first two terms in the right-hand side of (2.15) go to zero as $\varepsilon \rightarrow 0$. Hence (2.7) follows by Gronwall's inequality. Convergence (2.9) follows easily from (2.7) and (2.14) and standard arguments.

We remark that $M_{\varepsilon}(t)$ does not follows for $\varepsilon>0$ a particle path. Particle paths become, in the limit $\varepsilon \rightarrow 0$, more and more singular, and there is no hope to control them.

\section{3. $N$ Vortices}

We consider $N$ blobs of vorticity initially supported in $N$ disjoint open regions $\left\{\Lambda_{i}^{\varepsilon}\right\}_{i=1}^{N}$, such that meas $\Lambda_{i}=\varepsilon^{2}$ :

$$
\omega_{\varepsilon}(x, 0)=\sum_{i=1}^{N} a_{i} \varepsilon^{-2} X_{\Lambda_{i}^{\varepsilon}}(x), \quad a_{i} \in \mathbb{R} .
$$


We want to study the limiting behaviour of $\omega_{\varepsilon}(x, t)$, when $\varepsilon \rightarrow 0$, where $\omega_{\varepsilon}(x, t)$ is the solution of the Euler equations with initial datum (3.1).

As before, we introduce the centers of vorticity and the second moments of each blob

$$
\begin{gathered}
M_{\varepsilon}^{i}(t)=\varepsilon^{-2} \int_{\Lambda_{i, t}^{\varepsilon}} x d x, \\
I_{\varepsilon}^{i}(t)=a_{i} \varepsilon^{-2} \int_{\Lambda_{i, t}^{i}}\left(x-M_{\varepsilon}^{i}(t)\right)^{2} d x,
\end{gathered}
$$

where $\Lambda_{i, t}^{\varepsilon}$ is the evolution of the region $\Lambda_{i}^{\varepsilon}$ under the Euler flow, whose characteristics satisfy

$$
\left.\begin{array}{l}
\dot{x}^{\varepsilon}\left(t, x_{0}\right)=u_{\varepsilon}\left(x^{\varepsilon}\left(t, x_{0}\right), t\right), \quad x_{0} \in \Lambda_{i}^{\varepsilon}, \quad x^{\varepsilon}\left(0, x_{0}\right)=x_{0}, \\
u_{\varepsilon}(x, t)=\varepsilon^{-2} \sum_{i=1}^{N} a_{i} \int_{\Lambda_{1, t}^{\varepsilon}} k(x-y) d y .
\end{array}\right\}
$$

We notice that the above evolution is so regular that the region $\Lambda_{i, t}^{\varepsilon}$ never overlaps due to the uniqueness of characteristics.

It is convenient to introduce a regularized Euler dynamics and the corresponding vortex model in the following way. The characteristics of the regularized Euler motion satisfy:

$$
\left.\begin{array}{l}
\dot{\tilde{x}}^{\varepsilon}\left(t, x_{0}\right)=\tilde{u}_{\varepsilon}\left(\tilde{x}^{\varepsilon}\left(t, x_{0}\right), t\right), \quad x_{0} \in \Lambda_{i}^{\varepsilon}, \quad \tilde{x}^{\varepsilon}\left(0, x_{0}\right)=x_{0}, \\
\tilde{u}_{\varepsilon}(x, t)=a_{i} \varepsilon^{-2} \int_{\tilde{\Lambda}_{i, t}^{\varepsilon}} k(x-y) d y+\varepsilon^{-2} \sum_{j \neq i} a_{j} \int_{\tilde{A}_{J, t}^{\varepsilon}} k_{\eta}(x-y) d y,
\end{array}\right\}
$$

where $K_{\eta}$ is a smooth version of $k$ and $\left\{\tilde{\Lambda}_{i, t}^{\varepsilon}\right\}_{i=1}^{N}$ are the images at time $t$ of the initial regions $\Lambda_{i}^{\varepsilon}$ under the motion whose characteristics are given by (3.5).

We define

$$
\begin{gathered}
K_{\eta}(x)=\nabla^{\perp} g_{\eta}(x), \quad g_{\eta}(x)=\tilde{g}_{\eta}(|x|), \\
\tilde{g}_{\eta}(|\cdot|) \in C^{\infty}\left(\mathbb{R}^{1}\right), \quad \tilde{g}_{\eta}(|r|)=-\frac{1}{2 \pi} \ln |r| \quad \text { if } \quad|r| \geqq \eta, \\
\left|\frac{\partial}{\partial x_{i}} K_{\eta}(x)\right| \leqq \frac{c}{\eta^{2}} \quad x=\left(x_{1}, x_{2}\right), \quad i=1,2
\end{gathered}
$$

(from now on $C$ stands for a positive constant). We denote by $\tilde{M}_{\varepsilon}^{i}(t)$ and $\tilde{I}_{\varepsilon}^{i}(t)$ the same quantities as (3.2) and (3.5) with $\Lambda_{i, t}^{\varepsilon}$ replaced by $\tilde{\Lambda}_{i, t}^{\varepsilon}$.

The limiting vortex model related to $(3.5)$ is :

$$
\left.\begin{array}{l}
\dot{\tilde{x}}_{i}(t)=\sum_{\substack{j=1 \\
j \neq 1}}^{N} a_{j} K_{\eta}\left(\tilde{x}_{i}(t)-\tilde{x}_{j}(t)\right) \\
\tilde{x}_{i}(0)=x_{i}
\end{array}\right\}
$$

As a straightforward consequence of the previous section we have:

Theorem 3.1. Suppose

$$
\lim _{\varepsilon \rightarrow 0} \int f(x) \omega^{\varepsilon}(x, 0) d x=\sum_{i=1}^{N} a_{i} f\left(x_{i}\right)
$$


for any continuous, bounded function $f: \mathbb{R}^{2} \rightarrow \mathbb{R}$. Then for all $t>0$

$$
\lim _{\varepsilon \rightarrow 0} \tilde{M}_{\varepsilon}^{i}(t)=\tilde{x}_{i}(t)
$$

and

$$
\lim _{\varepsilon \rightarrow 0} \int f(x) \tilde{\omega}^{\varepsilon}(x, t) d x=\sum_{i=1}^{N} a_{i} f\left(\tilde{x}_{i}(t)\right)
$$

where

$$
\tilde{\omega}_{\varepsilon}(x, t)=\sum_{i=1}^{N} a_{i} \varepsilon^{-2} X_{\tilde{\Lambda}_{i, t}^{e}}(x) \text {. }
$$

Proof. Proceeding as in Theorem (2.1), for all $i \in\{1 \ldots N\}$,

$$
\left|\tilde{I}_{\varepsilon}^{i}(t)\right| \leqq\left|I_{\varepsilon}^{i}(0)\right| e^{\frac{c}{\eta^{2}} t}
$$

Rephrasing (2.15):

$$
\begin{aligned}
\left|\tilde{M}_{\varepsilon}^{i}(t)-\tilde{x}_{i}(t)\right| \leqq & \left|M_{\varepsilon}^{i}(0)-x_{i}\right| \\
& +\int_{0}^{t} d s\left|\sum_{\substack{j \neq 1 \\
j \neq 1}}^{N} \varepsilon^{-2} a_{j} \int_{\tilde{\Lambda}_{j, s}^{\epsilon}}\left[K_{\eta}\left(\tilde{M}_{\varepsilon}^{i}(t)-y\right)-K_{\eta}\left(\tilde{x}_{i}(s)-y\right)\right] d y\right| \\
& +\int_{0}^{t} d s\left|\sum_{\substack{j=1 \\
j \neq 1}}^{N} \varepsilon^{-2} a_{j} \int_{\tilde{A}_{j, s}}\left[K_{\eta}\left(\tilde{x}_{i}(s)-y\right)-K_{\eta}\left(\tilde{x}_{i}(s)-x_{j}(s)\right)\right] d y\right| \\
\leqq & \left|M_{\varepsilon}^{i}(0)-x_{i}\right|+\int_{0}^{t} d s \frac{(N-1) a c}{\eta^{2}}\left|\tilde{M}_{\varepsilon}^{i}(s)-\tilde{x}_{i}^{\varepsilon}(s)\right| \\
& +\int_{0}^{t} d s \frac{c}{\eta^{2}} \sum_{\substack{j=1 \\
j \neq 1}}^{N} \varepsilon^{-2}\left|a_{j}\right| \int_{\Lambda_{j, s}^{\varepsilon}}\left|y-\tilde{M}_{\varepsilon}^{j}(s)\right| d y \\
& +\int_{0}^{t} d s \frac{c}{\eta^{2}} \sum_{\substack{j=1 \\
j \neq i}}^{N}\left|a_{j}\right|\left|\tilde{M}_{\varepsilon}^{j}(s)-\tilde{x}_{j}(s)\right|,
\end{aligned}
$$

where $a=\max _{1 \leqq i \leqq N}\left|a_{i}\right|$. Applying the Cauchy-Schwarz inequality in the $2^{\text {nd }}$ term of the right-hand side of (3.13), and using (3.12), summing on $i$, the proof is easily completed by Gronwalls lemma.

Now we prove the main result of this paper

Theorem 3.2. Suppose (3.8) holds and in addition:

$$
\begin{gathered}
\min _{i \neq j} \inf \left\{|x-y| \mid x \in \Lambda_{i}^{\varepsilon}, y \in \Lambda_{j}^{\varepsilon}\right\}=R \geqq \frac{1}{2} \min _{i \neq j}\left|x_{i}-x_{j}\right|>0, \\
\left|I_{\varepsilon}^{i}(0)\right| \leqq C_{i} \varepsilon^{2}
\end{gathered}
$$


for some $C_{i}>0$. Then, there exists a time $T$, such that, for all $t \in[0, T)$

$$
\lim _{\varepsilon \rightarrow 0} M_{\varepsilon}^{i}(t)=x_{i}(t),
$$

where $\left\{x_{1}(t) \ldots x_{N}(t)\right\}$ solve the initial value problem (1.4). Moreover

$$
\lim _{\varepsilon \rightarrow 0} \int f(x) \omega^{\varepsilon}(x, t) d x=\sum_{i=1}^{N} a_{i} f\left(x_{i}(t)\right) .
$$

Remark. Condition (3.1) can be easily realized supposing $\Lambda_{i}^{\varepsilon}$ contained in a circle centered in $x_{i}$ of radius of the same order of $\varepsilon$.

Proof of Theorem 3.2. We first notice that the regularized vortex motion (3.7) coincides with the real vortex motion up to the time $T_{\eta} \inf \left\{t\left|\min _{i, j}\right| \tilde{x}_{i}(t)-\tilde{x}_{j}(t) \mid \leqq \eta\right\}$. Furthermore the regularized Euler dynamics coincides with the real Euler dynamics up to the time

$$
T_{\eta}^{\varepsilon}=\inf \left\{t \mid \min _{i \neq j} \operatorname{dist}\left(\Lambda_{i, t}^{\varepsilon}, \Lambda_{j, t}^{\varepsilon}\right) \leqq \eta\right\}
$$

Hence Theorem 3.2 is proved by using Theorem 3.1 once one shows the existence of a positive $\eta$ for which $\min \left(T_{\eta}, \inf T_{\eta}^{e}\right)>0$.

Given $x_{1} \ldots x_{N}$, one can find a time $T^{*}$ for which $\min _{i \neq j} \inf _{0 \leqq t \leqq T^{*}}\left|x_{i}(t)-x_{j}(t)\right| \geqq b$ $>0$, and hence, choosing $\eta=b \backslash 3, \tilde{x}_{i}(t)=x_{i}(t)$ for $t \in\left[0, T^{*}\right)$.

Let $\Sigma_{d, i}^{\varepsilon}(t)$ be the circle of radius $d$ around $\tilde{M}_{\varepsilon}^{i}(t)$. Then defining

$$
\tilde{\Lambda}_{i, t}^{\varepsilon} \cap \mathbb{R}^{2} / \Sigma_{d, i}^{\varepsilon}(t)=A_{i}^{\varepsilon}(t),
$$

we have, by (3.12) and (3.14b), and for $t<T^{*}$,

$$
\left|a_{i}\right| d^{2} \varepsilon^{-2} \operatorname{meas} A_{i}^{\varepsilon}(t) \leqq\left|\tilde{I}_{\varepsilon}^{i}(t)\right| \leqq C_{i} \varepsilon^{2} e^{\frac{C}{\eta^{2} t}} .
$$

Hence the velocity field generated by the vorticity supported in $A_{i}^{\varepsilon}(t)$ is bounded by

$$
\left|a_{i}\right| \varepsilon^{-2} \sup _{x} \int_{A_{i}^{\varepsilon}(t)} \frac{1}{|x-y|} d y \leqq\left|a_{i}\right| \varepsilon^{-2} \int_{B_{i}^{\varepsilon}(t)} \frac{1}{|y|} d y,
$$

where $B_{i}^{\varepsilon}(t)$ is the circle of radius $\sqrt{\text { meas } A_{i}^{\varepsilon}(t) \backslash \pi}$. Therefore, the right-hand side of (3.20) is bounded by

$$
\frac{K_{1}}{d} \exp \frac{C}{\eta^{2}} T^{*}
$$

where $K_{1}$ is a positive constant. The velocity field outside the region $\sum_{2 d, i}^{\varepsilon}(t)$ is certainly bounded by

$$
\frac{\left|a_{i}\right|}{2 \pi d}+\sum_{\substack{j=1 \\ j \neq i}}^{N} \frac{\left|a_{j}\right|}{\eta}+\frac{K_{1}}{d} e^{\frac{c}{\eta^{2}} T^{*}},
$$

where the first contribution is a bound for the velocity field generated by the vorticity inside $\Sigma_{i, d}^{\varepsilon}(t)$, the second is due to the other blobs, and finally the last is due to the vorticity outside $\Sigma_{i, d}^{\varepsilon}(t)$. 
Finally, choosing $\varepsilon$ so small that $\sup _{i} \sup _{t \in\left[0, T^{*}\right]}\left|\tilde{x}_{i}(t)-\tilde{M}_{i}^{\varepsilon}(t)\right|<\frac{d}{2}$, and putting $d=b \backslash 100$, we conclude that for $\varepsilon$ sufficiently small,

$$
\begin{aligned}
& \min _{i \neq j} \inf _{s \leqq t} \operatorname{dist}\left(\tilde{\Lambda}_{i, s}^{\varepsilon}, \tilde{\Lambda}_{j, s}^{\varepsilon}\right) \\
& \geqq \frac{b}{2}-5 d-2 t\left(\frac{a}{2 \pi d}+\frac{N-1}{\eta} a+\frac{K_{1}}{d} e^{\frac{c}{\eta^{2}} T^{*}}\right),
\end{aligned}
$$

where $a=\max _{i}\left|a_{i}\right|$. Hence one can choose $t$ so small that the right-hand side of (3.23) is larger than $\eta=b / 3$.

We conclude the section with some comments.

We have shown a rigorous connection between Euler equations and vortex theory by proving that the selfenergy is negligible at least for short times. In general, it is the best one can prove without further assumptions on the initial distribution of the vorticity $\sum_{i=1}^{N} a_{i} \delta_{x_{i}}(d x)$. In fact it has been proved that the vortex motion can produce collapses in finite time [4]. Nevertheless it has also been proved that the initial conditions giving rise to singularities in finite times have Lebesgue measure zero (see $[5,3]$ for bounded and more general domains, respectively). Thus the full Lebesgue measure set of initial vortex configurations for which the motion is regular, the above approximation in terms of the Euler flow is, at least, conceivable for all times. On the other hand, numerical simulations on the so-called contour dynamics (the Euler evolutions for step functions we have investigated in this paper) seem to show that the boundaries of the regions $\Lambda_{i, t}^{\varepsilon}$ (contours) some regularity in finite time. Thus it may be difficult to prove for all time our result.

We finally remark we need an extra condition for the initial blobs [Condition (3.14b)] in Theorem 3.2, while weaker conditions were sufficient for Theorems 2.1 and 3.1. Nevertheless it has been shown that it is not necessary to assume a complete symmetry of the vorticity profile around the center $x_{i}$ 's, whereas it is used in some textbooks to justify the negligibility of the self-interaction.

Now we discuss the case when the domain $D$ in which the fluid is confined, is simply connected and boundaries are present.

In this case the vortex model takes the form:

$$
\left.\begin{array}{l}
\dot{x}_{i}(t)=\sum_{\substack{j=1 \\
j \neq i}}^{N} a_{i}\left(\nabla_{x_{i}}^{\perp} g_{D}\right)\left(x_{i}, x_{j}\right)+\frac{1}{2} a_{i} \nabla_{x_{\imath}}^{\perp} \gamma\left(x_{i}, x_{i}\right), \\
x_{i}(0)=x_{i},
\end{array}\right\}
$$

where $g_{D}$ is the Green function of the Laplace operator with Dirichlet boundary conditions and

$$
\gamma(x, y)=g_{D}(x, y)+\frac{1}{2 \pi} \ln |x-y| .
$$

The term $\gamma\left(x_{i}, x_{i}\right)$ in Eqs. (3.24) takes into account the interaction of the $i$-vortex with the boundary. 
The Euler evolution takes the same form as (3.4) replacing $k$ by $\nabla^{\perp} \mathrm{g}_{D}$.

All our previous analysis can be carried out in this case also, and an analogue of Theorem 3.2 can be proved along the same lines, taking into explicit account the interaction of the blobs with the boundaries in the approximate conservation law for $M_{\varepsilon}$ and $I_{\varepsilon}$. It gives rise to the extra term $\frac{1}{2} a_{i} \nabla_{x_{i}}^{\perp} \gamma$ in the right-hand side of (3.24).

Acknowledgements. One of us (C.M.) thanks very much J. Lebowitz and E. Lieb for the hospitality at the Math. Dept. of Rutgers University and Phys. Dept. of Princeton University, where part of this reasearch was carried out.

\section{References}

1. Helmholtz, H.: On integrals of hydrodynamical equations which express vortex-motion. Philos. Mag. 33, 485 (1867)

Kirchoff, G.R.: Vorlesungen über Math. Phys., Vol. I. Leipzig: Teubner 1876

Poincaré, H.: Theorie des turbillons. Paris: G. Carré 1893

2. Hald, O., Mauceri Del Prete, V. v.: Convergence of vortex methods for Euler's equations. Math. Comp. 32, 791 (1978)

Hald, O.: The convergence of vortex methods. II. SIAM J. Numer. Anal. 16, 726 (1979)

Marchioro, C., Pulvirenti, M.: Hydrodynamics in two-dimensions and vortex theory. Commun. Math. Phys. 84, 483 (1982)

Beale, J.T., Majda, A.: Vortex methods. I. Convergence in three-dimensions. Math. Comp. 39, 1 (1982); Vortex methods. II. Higher order accuracy in two- and three-dimensions 39, 29 (1982)

3. Marchioro, C., Pulvirenti, M.: Vortex methods in two-dimensional fluid dynamics (in preparation) (1983)

4. Aref, H.: Motion of three vortices. Phys. Fluids 22, 393 (1979)

5. Dürr, D., Pulvirenti, M.: On the vortex flow in bounded domains. Commun. Math. Phys. 85, 256 (1982)

Communicated by A. Jaffe

Received July 6, 1983 